\title{
JAHRBÜCHER
}

FUR

\section{NATIONALÖKONOMIE UND STATISTIK}

GEQRUN DET VON

BRUNO IILDEBRAND.

HERAUSGEGEBEN VON

DR. J. CONRAD, und DR. L. ELSTER,

PROP. IM GALLE A. B., PHOP. IH BRERLU,

IN VERBINDUNG MIT

DR. EDG. LOENING, und DR. W. LEXiS,

PEor. IM gaLLx 4. B., PRor. IN abtTrmary.

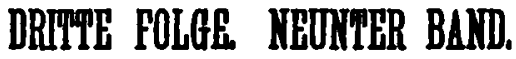

ERSTE FOLGE, BAND I-XXXIV; ZWEITE FOLGE, BAND XXXV-LV ODEI NEOE FOLGE, BAND I-XXI; DRITTE FOLGE, BAND LXIV (III. FOLGE, BAND IX).

Reprint

SCHMIDT PERIODICALS GMBH

D-8201 Bad Feilnbach 2 / W. Germany 
Nachdruck mit Genehmigung des Gustav Fischer Verlags GmbH \& Co. KG., Stuttgart.

Die Vorlagen für den Nachdruck stellte die Universitätsbibliothek Tübingen freundlicherweise zur Verfügung. 


\title{
JAHRBÜCHER
}

FUR

\section{NATIONALÖKONOMIE UND STATISTIK}

\author{
GEGRUN DET VON \\ BRUNO HLDEBRAND.
}

HERAUSGEGEBEN VON

DR. J. CONRAD, uND DR. L. ELSTER,

PBOT. IH BALLE 4. B., PROT. IX BREsLAd,

IN VERBINDUNG MIT

DR. EDG. LOENING, UND DR. W. LEXIS,

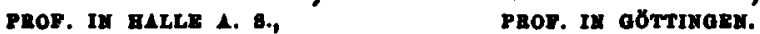

\section{DRIIPR POLGE NEOAPER BAND.}

ERSTE FOLGE, BAND I-XXXIV, ZWBITE FOLGE, BAND XXXV-LV ODEK NEUE FOLGE, BAND I-XXI; DRITTE FOLGE, BAND LXIV (III. FOLGE, BAND IX).

J E N A,

VERLAG VON GUSTAV FISOHER.

1895 . 
